\title{
Examination of Nonlinear Distortion of IT Power Supplies in Low Voltage Grid from the Current Quality Point of View
}

\author{
V. SzÜCS, A. Fodor, A. GÖllei AND P. GÖRBE*
}

University of Pannonia, Department of Electrical Engineering and Information Systems, 8200 Veszprem, Hungary

\begin{abstract}
This work is devoted to investigating the current nonlinear distortion of IT devices linear and switching mode power supplies. A new developed software package is shown suitable for separate varying degrees load of functional parts of personal computers. Based on the collected voltage and current harmonic analysis serious distortion is stated in current time functions in automatic power factor compensating devices, too. Enormous distortions are observed in simple structure power supplies low power consumption consumer electronic devices. The consequences and effects of this behavior is described, and possible solutions are adumbrated in detailed conclusion with integration of domestic size complex energetic systems consisting of renewable energy sources, battery storage and single and three phase current inverter parts, especially in development of direct current bus systems in computer system applications.
\end{abstract}

DOI: 10.12693/APhysPolA.134.307

PACS/topics: renewable energy, nonlinear distortion, DC grid technology

\section{Introduction}

Nowadays the using of renewable energy sources in island mode [1] and grid connected distributed generation are spreading in domestic size applications, too. This can be combined with energy storage devices, for example batteries of electrical vehicles, to reduce the stochastic behavior of generally exploited energy of photovoltaic and wind turbine applications [2-4]. The rising number of IT and consumer electronic devices (mobile phone chargers, tablet and notebook power supplies, switching mode power supplies (SMPS) in personal computers and server configurations, televisions, inverter air condition devices, LED lighting) cause serious, previously not seen, nonlinear distortion in low voltage grid applications. It has undesirable effects observed in low voltage transformer areas, and in significantly high distorted cases in high voltage parts of the electrical energy distributing systems, too. This distortion is increasing the overall power loss of the whole grid system, increasing the carbon footprint of our everyday life, causing higher green house gases (GHG) emission to accelerate global warming and climate change.

The nonlinear distortion can be formulated with total harmonic distortion (THD) (Eq. (1)), where $V_{k}$ is the $k$-th harmonic component amplitude of the voltage and $V_{1}$ is the base harmonic component amplitude value. This definition is suitable for description of current distortion too in the similar definition:

$$
\mathrm{THD}=\sqrt{\frac{\sum_{k=2}^{\infty}\left(\left|V_{k}\right|^{2}\right)^{2}}{V_{1}}}
$$

*corresponding author; e-mail: gorbep@almos.uni-pannon.hu
The distorted current shape raises the power loss of line conductors compared to the ideal linear sinusoid case (Eq. (2)), where $P_{p h d}$ is the raised power loss of the line wire, $P_{\text {phid }}$ is the power loss in ideal sinusoid case and THD is the total harmonic distortion of the current

$$
P_{\mathrm{ph} \mathrm{d}}=P_{\mathrm{ph} \mathrm{id}}\left(1+\mathrm{THD}^{2}\right) \text {. }
$$

Greater additional power loss presumable on zero-line wires, because in ideal symmetric case the current effective value is minimal on it. On the other aspect, the expected odd harmonic components are of hazardous types, because the upper harmonic components are not cancelled out but mathematically added up, if the upper harmonic index can be divided into three. The additional power loss can be estimated (Eq. (3)).

$$
P_{\text {ne d }}=P_{\text {ne id }} 9 \mathrm{THD}^{2} \text {, }
$$

where $P_{\text {ne d }}$ is the raised power loss of the neutral or zero line, $P_{\text {ne id }}$ is the power loss in ideal sinusoid case and THD is the total harmonic distortion of the current. These additional losses are raising the operational temperature of the wires, in serious distorted case this can cause fire in neutral lines. Other additional undesirable effects are caused by nonlinear distortion, too:

- Skin effect of higher frequency components;

- Protection circuit undesired operation;

- Resonance with power factor compensating capacities;

- Overloading of transformers.

These additional carbon footprints are negligible, but reduction in operational reliability can cause a significant increase in costs.

With the help of method developed in the authors earlier researches, the prepared solution was able to reduce the described effects significantly. By injecting upper harmonic current component into the low voltage grid 
using complex energetic solution in one device to collect the electric energy from renewable source, balance the stochastic high and low peaks of power production and consumption with charging and discharging connected batteries in MATLAB Simulink simulations $[5,6]$.

\section{IT solution for THD measurement based on managed, linear, scalable load}

During this examination process the authors developed a special load simulation software (SLS), suitable to manage the scalable, linear load on notebooks, personal computers and the second module of this software can be used to measure the real-time changes in the current, voltage and power of the electric connection in time end frequency domain and log the grid's power parameters in every second.

The SLS software gives an excellent opportunity to discover the separate effects of the main functional parts of these computer systems to the power quality through the energy transportation lines in buildings via the examination of total harmonic distortion correlated to the current power load. The main functions are: the setting of the CPU load or overload per core, setting the memory load, HDD load, and graphical processor unit (GPU) load parameters. In the SLS software, the user can predefine some special load-profiles and can run these or can set a unique parameter set to load the hardware, as can be seen in Fig. 1.

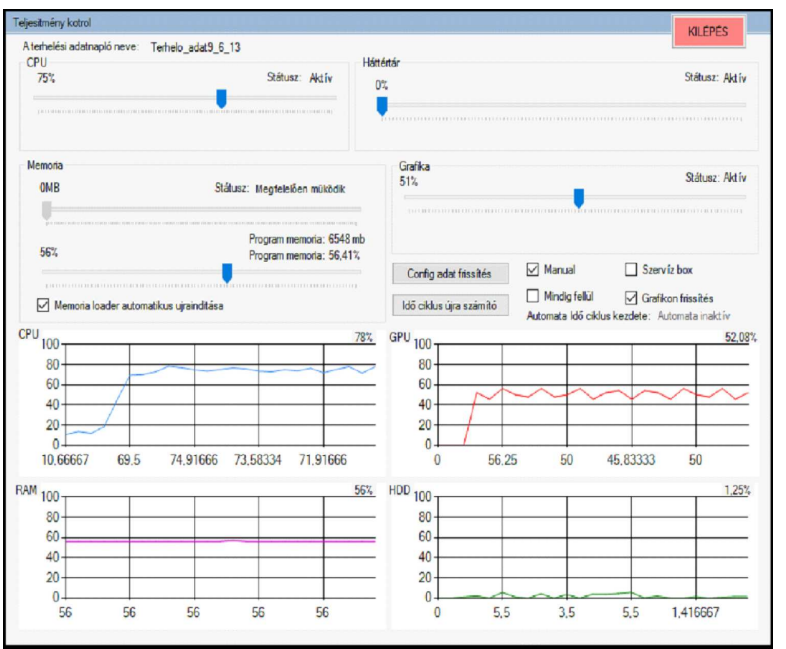

Fig. 1. Load control display in SLS software.

During the measurement, the SLS software continually adjusts the convenient load on laptops so that the greatest possible measurement area using with optimal step. The tests and measurements which were performed with the use of SLS software were logged to database continuously.

A data-logger module is built in the SLS software as well. This module can test the serial connection between the computer and the measurement device; can check the communication port settings, and using this module the user can set the properties for measurement.

Several measurements were performed on different IT devices, on personal computers and on notebooks. The results show that in any cases the harmonic distortion is at unexpected high level. The value and the range of THD were between $110 \%$ and $180 \%$. The most significant THD values were registered on notebooks power supplies, the other mobile devices generated a smaller THD on the grid.

The effects of nonlinear electric load can be more visualized via the Fourier coefficients in every time period in the frequency domain. During the measurements, the authors found some interesting details, as can be seen in Fig. 2. The current input value on even harmonics is different from zero, and on odd harmonics, huge THD values appeared. It is a very dangerous phenomenon on low voltage grids, and this can generate some undesirable effects and can eventuate a significant power loss and radical carbon footprint rising.

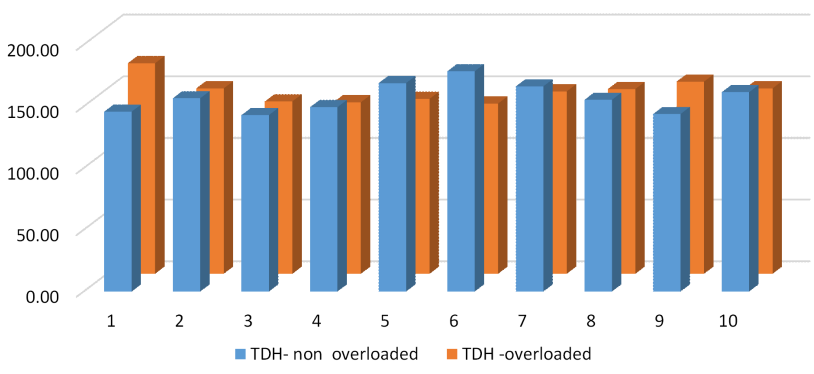

Fig. 2. THD values on 10 measures.

The evaluation of the measured values proved that the power quality can be improved though, the supposed total harmonic distortion in many cases is much higher value than expected, the upper harmonic components are in the same scale as the base harmonic current value for single-phase loads as can be seen in Fig. 3 .

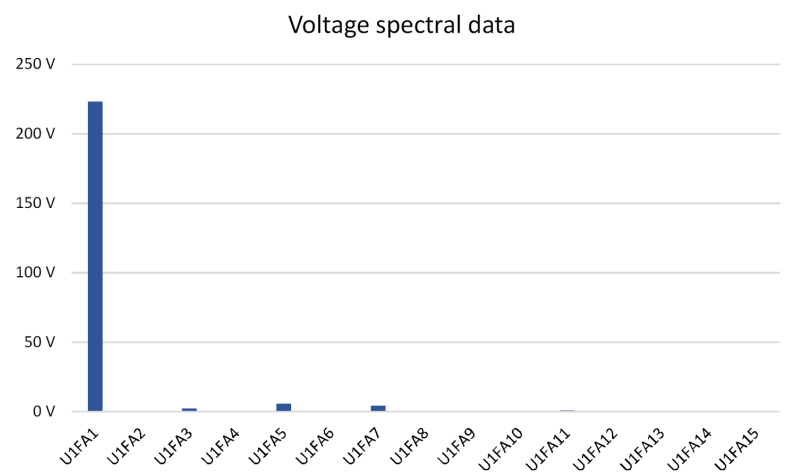

Fig. 3. Voltage spectral data in our laboratory $\left(\mathrm{THD}_{U}\right.$ $3.35 \%)$.

During data analysis, there was found unexpected phenomenon in current frequency domain. Although the examined personal computer (PC) uses automatic power factor correction (APFC) SMPS inside we expected a 
nearly linear resistive behavior from AC connection side. The same condition was expected in the spectral amplitude dispersion regarding to the voltage and current as well. The voltage spectra show small scale nonlinear distortion in building's grid area (Fig. 3). The measured notebook supply shows serious distortion in current shape because of its low cost simplified structure non-APFC construction (just half or full bridge rectifying with capacitor for DC conversion at first stage), see in Fig. 4.

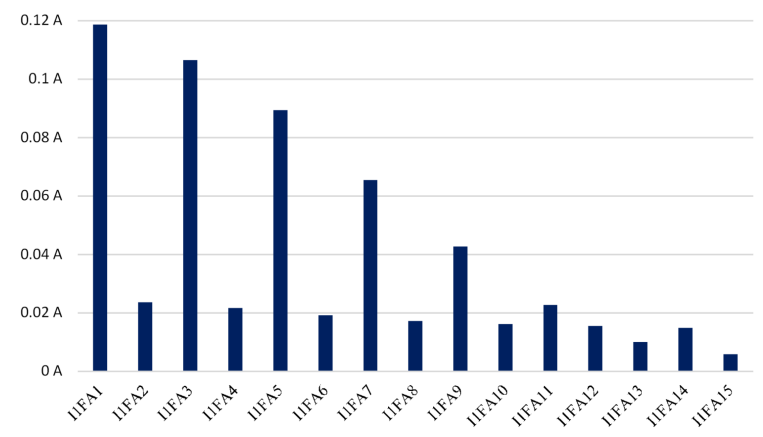

Fig. 4. Current spectral data, notebook power supply $\left(\mathrm{THD}_{I} 142.31 \%\right)$.

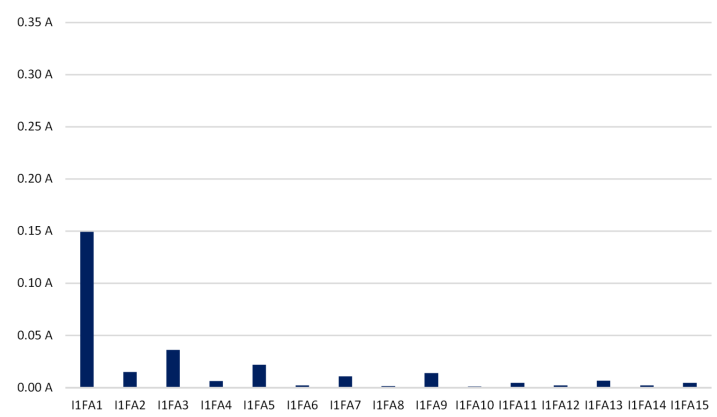

Fig. 5. Current spectral data: $\mathrm{PC}$ on $15 \mathrm{~W}$ load $\left(\mathrm{THD}_{I}\right.$ $33.22 \%$ ).

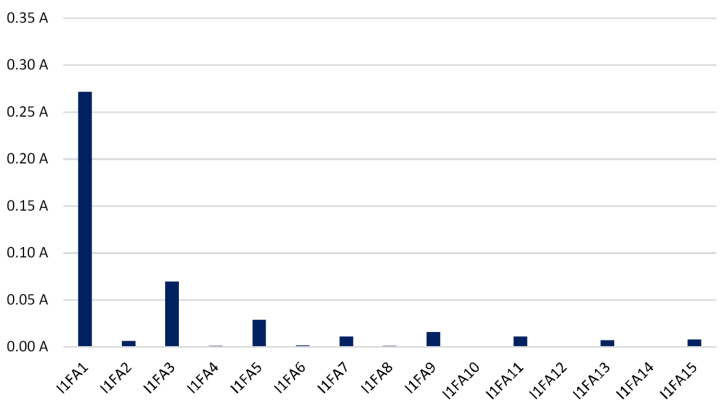

Fig. 6. Voltage spectrum $\mathrm{PC}$ on $52 \mathrm{~W}$ load $\left(\mathrm{THD}_{I}\right.$ $29.22 \%$ ).

Serious distortion in current shape because it simple construction (Fig. 4). But the PC SMPS collected data shows serious distortion also (Fig. 5) and its spectral behavior is changing against the load value (on $52 \mathrm{~W}$ load

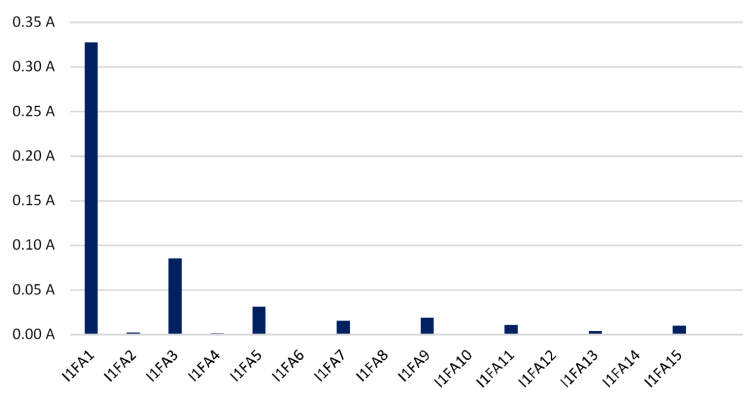

Fig. 7. Current spectral data: PC $92 \mathrm{~W}$ load $\left(\mathrm{THD}_{I}\right.$ $29.01 \%)$.

as can be seen in Fig. 6 and on $92 \mathrm{~W}$ load can be seen in Fig. 7). The appearance of large scale second upper harmonic component is unexpected. This type of nonlinear SMPS should generate mainly odd upper harmonic components. The minimal load situation shows weakness in this construction of SMPS from the power quality point of view. Unfortunately, most of the operational time of these points of the operating characteristics of these devices are specific.

\section{Solution for nonlinear distortion}

Low voltage DC grids can be complemented by batteries to store energy when the renewable sources are not able to supply the grid. A polynomial relationship between the exact charging state and the connection point voltage values can describe the whole storage system accurately. From the obtained experimental data, the surface diagram can be built (Fig. 8). The measurement was applied in specific points, but the polynomial surface diagram which was created with an interpolation technique can give adequate information about the whole system behavior. The deviation of the surface temperature of the cell from the environmental temperature well describes the efficiency during the charging and discharging process. With the help of this relationship, it is possible to build model-based simulations of battery behavior in different thermal conditions. It would be useful in the case of temperature-controlled quick battery charger application or in an application for an electrical vehicle in very extreme temperature conditions.

To use batteries for low voltage grids or electric vehicles it is important to know its behavior during charging and discharging procedure in different temperature. A temperature-dependent model for a family of batteries has been developed for vehicle electronics applications. The model of battery was built from obtained measurement data. The battery model has been verified by simulation as well in a complex energetic system. The energetic system and battery model was implemented in the Matlab/Simulink environment. With the help of this temperature dependent model charge estimation, state of charge, state of health can be calculated regarding to the actual battery. It is useful for example to estimate remaining distance in an electric car or to estimate the bridging capability of blackout in a low voltage DC line. 


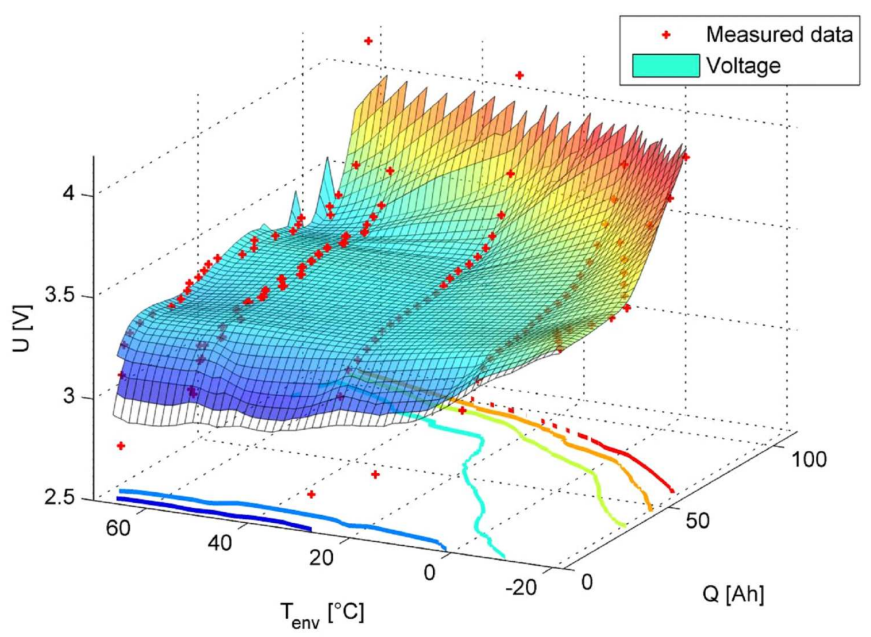

Fig. 8. The polynomial surface of a battery voltage.

The proposed measurement method and modeling setup is commonly used, it is applicable to any electrical (both of linear and nonlinear) two pole system.

During the examination, the using of electricity for a building using small power plants, then it can be determined that most electronic devices do not operate directly from AC sinusoidal voltage. These devices convert the AC supply to DC operating voltage externally or internally. In such cases, such as DC voltage produced by the solar panels is fed using an inverter to the electric AC grid, and then a few meters next to the entry points a lot of electrical devices convert the AC voltage to $\mathrm{DC}$ voltage.

The basic idea of the DC micro-grid network is to save the electrical power conversion losses and this idea can solve a lot of existing problems of the $\mathrm{AC}$ electrical grid. Unfortunately, in this case, the extra expense would be shown to the cabling costs and the losses of the DC grid voltage regulation.

However, this method provides an opportunity for simplification of the power supply of a lot of devices and a lot of devices could increase their efficiency as well.

The battery chargers and those batteries are connected to the DC grid, with this method the uninterruptible power supplying is also much easier and more efficiently feasible than the current UPS architectures, because instead of converting two (AC/DC and $\mathrm{DC} / \mathrm{AC}$ ) would require only a $\mathrm{DC} / \mathrm{DC}$ converter. If you want to apply this solution for computers and servers, it just needs to be done to the power supply that it must be replaced with a $\mathrm{DC} / \mathrm{DC}$ converter.

Because of it there is conversion loss in any case, one conversion of energy can be saved by skipping one converter and we can increase system efficiency. This efficiency increase is important in that case when the system is operating from battery because back-up time is growing.

The using of DC grid technology has begun with industrial applications using in parallel with the $\mathrm{AC}$ voltage networks, these systems powered by the AC power supply in parallel with renewable DC grid in cases where consumer demand is greater than the renewable generated electricity [7].

Using the DC micro-grid energy generation and supply of electricity to consumers would be undisturbed for external power failure. In this case AC power injection must be disabled and the energy balance must be made, too. A further advantage is that low energy DC consumers do not pollute the $\mathrm{AC}$ network with their harmonic currents because it would be operated from the DC grid.

In this work the current nonlinear behavior of IT SMPS loads were investigated. A new developed software package had been shown suitable for controlled changing load of functional parts of PCs. Based on the collected voltage and current harmonic analysis serious distortion has been found in APFC mode devices, too. Enormous distortions have been observed in simple structure power supplies, low power consumption notebook computers.

The experienced serious distortion extrapolates serious growth in power loss and carbon footprint and extrapolates serious growth in transportation lines and transformers malfunction and middle term blackouts, too.

\section{Conclusions}

The decreasing of this distortion is inevitable with upper harmonic current injection from renewable sources or battery storages (for example grid connected EVs) from DG form, or radical improvement of SMPS constructions, or DC based microgrid spreading in households and IT server farms as well.

\section{Acknowledgments}

The authors would like to tell a special thanks to their colleagues, who participated in the SLS software development process, to Mr. Gyula Szabó and Daniel Hruby for measurements' data.

The authors acknowledge the support of the Hungarian Government's National Research, Development and Innovation Office - NKFIH through grant no. SNN120422.

\section{References}

[1] O. Kisseleva, B. Akhmetov, P. Kharitonov, A. Engelko, H. Bluhm, Acta Phys. Pol. A 128, B-258 (2015).

[2] A.A. Jadallah, D.Y. Mahmood, Z. Er, Z.A. Abdulqader, Acta Phys. Pol. A 130, 434 (2016).

[3] Z. Er, Acta Phys. Pol. A 130, 72 (2016).

[4] A.A. Jadallah, D.Y. Mahmood, Z.A. Abdulqader, Acta Phys. Pol. A 128, B-461 (2015).

[5] P. Görbe, A. Magyar, K.M. Hangos, J. Cleaner Product. 34, 125 (2012).

[6] A. Göllei, P. Görbe, A. Magyar, J. Cleaner Product. 34, 138 (2012).

[7] N. Ayai, T. Hisada, T. Shibata, H. Miyoshi, T. Iwasaki, K. Kitayama, Electric Wire Cable Energy 25, 132 (2014). 